\title{
Ausencia de diferencias entre los accesos radial y femoral durante las intervenciones percutáneas coronarias. Resultados a 30 días de un registro prospectivo y consecutivo de pacientes
}

\author{
Absence of differences between radial and femoral access during \\ percutaneous coronary interventions. 30-day results of a prospective \\ and consecutive patient registry
}

Juan Mieres, Carlos Fernández-Pereira, Hernán Pavlovsky, Omar Santaera, Juan Del Pozo, Javier Mendoza, Francisco Peralta-Bazán, Matías A Rodríguez-Granillo, Santiago Burda, Yazmín Navarro, Alfredo E. Rodríguez

\section{RESUMEN}

En este registro prospectivo de pacientes con angioplastia donde se compara la vía radial con la femoral y sus resultados a 30 días en un solo centro en la Argentina durante un período de dos años con cardiólogos intervencionistas entrenados en ambos procedimientos, con un protocolo de punción, control y seguimiento por vía femoral. Analizamos la población global, sin shock cardiogénico y sin infarto agudo del miocardio, en forma directa y luego con un test de propensity score matching, no encontrándose diferencias significativas entre estas técnicas, tanto en los eventos adversos combinados como en muerte, stroke y complicaciones vasculares mayores. Se halló una diferencia numérica pero no significativa en eventos vasculares menores a favor del abordaje radial, que incluyen pseudoaneurismas con manejo conservador y hemorragias menores. Podemos concluir que ambas vías pueden utilizarse indistintamente con un grupo de cardiólogos altamente entrenados en ambos abordajes.

Palabras claves: acceso radial, acceso femoral, STEMI, sangrado, angioplastia coronaria, complicaciones en el sitio de punción, propensity score matching.

\begin{abstract}
In this prospective registry of patients with angioplasty where the radial and femoral pathways are compared and their results at 30 days in a single center in Argentina over a period of two years with interventional cardiologists trained in both procedures, with a puncture protocol, control and follow-up by femoral approach. We analyzed the global population, without cardiogenic shock and without acute myocardial infarction directly and then with a "propensity score matching" test, finding no significant differences between these different techniques, both in the combined adverse events and in death, stroke and major vascular complications. Finding a numerical difference but not significant in minor vascular events in favor of the radia approach, which include pseudoaneurysms with conservative management and minor hemorrhages. We can conclude that both pathways can be used interchangeably in a group of highly trained cardiologists in both approaches.
\end{abstract}

Key words: radial access, femoral access, STEMI, bleeding, coronary angioplasty, site puncture complications, propensity score matching.

Revista Argentina de Cardioangiología Intervencionista 2019;10(2):63-67. https://doi.org/10.30567/RACI/201902/0063-0067

\section{INTRODUCCIÓN}

El acceso de la intervención percutánea fue evolucionando de acuerdo con el desarrollo de esta. Se inició con la angiografía por las vías humeral y femoral, con la técnica de Sones y Judkins ${ }^{1}$, respectivamente, y luego pasó rápidamente a la vía femoral por donde se realizó la primera angioplastia coronaria percutánea $(\mathrm{PCI})^{2}$, donde sufrió el más grande desarrollo y la mayoría de los catéteres fueron desarrollados para tal fin, de hecho, en la Argentina observamos que las primeras intervenciones percutáneas se desarrollaron por vía femoral incluyendo pacientes con infarto agudo del miocardio con elevación del segmento ST (STEMI) ${ }^{3}$.

Fue Campeu quien inicia la era de angiografías diagnósticas por vía radial ${ }^{4}(\mathrm{VR})$ y Kiemeneij ${ }^{5,6}$ la comienza a utilizar y la estandariza para angioplastias coronarias. Rápidamente ganó terreno después de ensayos randomizados donde mostrará sus beneficios en comparación con la vía femoral (VF) convirtién-

\footnotetext{
1. Centro CECl, Buenos Aires, Rep. Argentina

$\square$ Correspondencia: Dr. Juan Mieres.jmieres@centroceci.com.ar

Los autores no declaran conflictos de intereses
}

Recibido: 13/03/2019| Aceptado: 25/04/2019 dose en algunos países fundamentalmente europeos como el acceso de elección siguiendo las recomendaciones de las guías de la Sociedad Europea de Cardiología 7 . Sin embargo, a pesar de esto, en los registros de EE.UU. el acceso femoral sigue siendo la vía de elección en el $70 \%$ de los casos.

El propósito de esta presentación es mostrar los resultados de ambos accesos, femoral (AF) y radial (AR), en un grupo consecutivo y prospectivo de pacientes con angioplastia coronaria.

\section{MATERIAL Y MÉTODOS}

El propósito de este trabajo es exponer los resultados de 30 días con ambos enfoques en una experiencia de los laboratorios de Cateterización Cardíaca y Hemodinamia del Centro de Estudios en Cardiología Intervencionista (CECI) de Buenos Aires, Argentina.

Desde enero del año 2016 hasta enero del 2018 se incluyeron en forma prospectiva en la base de datos del Centro CECI, Cardioangiología Intervencionista Sanatorio Otamendi y Sanatorio Las Lomas, sobre 2240 procedimientos realizados en dichos laboratorios de Hemodinamia, 988 pacientes ingresados en el registro, todos ellos tratados con PCI. Las razones de la exclusión de los 1252 restantes fueron: el tratamiento concomitante en otro territorio vascular o la reparación estructural concomitante o el tratamiento percutá- 
TABLA 1. Características basales clínicas y angiográficas.

\begin{tabular}{|l|c|c|c|}
\hline & $\begin{array}{c}\text { Femoral } \\
\%(n=702)\end{array}$ & $\begin{array}{c}\text { Radial } \\
(\mathrm{n}=286)\end{array}$ & $p$ \\
\hline Mujeres & $20,9(147)$ & $8,4(262)$ & $<0,001$ \\
\hline Edad (años) & $67,9 \pm 11.6$ & $63,8 \pm 10.0$ & $<0,001$ \\
\hline Hipertensión arterial & $78,5(551)$ & $67,5(93)$ & $<0,001$ \\
\hline Dislipemia & $75,6(530)$ & $71,7(205)$ & 0,19 \\
\hline Tabaquista & $16,1(113)$ & $19,9(57)$ & 0,14 \\
\hline Diabetes & $24,6(173)$ & $24,8(71)$ & 0,95 \\
\hline Antecedentes familiares & $13(91)$ & $11,5(33)$ & 0,54 \\
\hline Infarto previo & $24,9(175)$ & $19,2(55)$ & 0,05 \\
\hline Revascularización previa & $37,3(262)$ & $28,0(80)$ & 0,005 \\
\hline CRM previa & $8,0(56)$ & $1,7(5)$ & $<0,001$ \\
\hline Enfermedad vascular periférica & $5,7(40)$ & $7,0(20)$ & 0,44 \\
\hline ACV & $3,3(23)$ & $3,1(9)$ & 0,91 \\
\hline Mala función ventricular & $7,8(55)$ & $4,5(13)$ & 0,06 \\
\hline Insuficiencia renal crónica & $6,0(42)$ & $4,9(14)$ & 0,50 \\
\hline BMI>29 & $17,1(120)$ & $26,9(77)$ & $<0,001$ \\
\hline Shock cardiogénico & $2,1(15)$ & $0,3(1)$ & 0,03 \\
\hline Síndrome coronario agudo & $79,2(556)$ & $76,2(218)$ & 0,30 \\
\hline Infarto agudo con STEMI & $15,7(110)$ & $8,7(25)$ & 0,004 \\
\hline Enfermedad de múltiples vasos & $39,6(278)$ & $39,9(114)$ & 0,94 \\
\hline Enfermedad del tronco de Clno protegido & $7,8(55)$ & $3,8(11)$ & 0,023 \\
\hline Anticoagulación previa & $6,0(34)$ & $8,9(23)$ & 0,11 \\
\hline Antiagregación previa & $20,7(145)$ & $28,3(81)$ & 0,009 \\
\hline Prasugrel & $19,1(134)$ & $9,1(26)$ & $<0,001$ \\
\hline Ticagrelor & $31,3(220)$ & $52,8(151)$ & $<0,001$ \\
\hline Clopidogrel & $49,0(344)$ & $38,1(109)$ & 0,002 \\
\hline Inhibidores de IIb/Illa & $19.6(137)$ & $23,4(67)$ & 0.17 \\
\hline Stents & $1,85 \pm 0,9$ & $1,72 \pm 0,9$ & 0,06 \\
\hline BMS (n) & 887 & 345 & \\
\hline DES (n) & 1409,8 & $182,74 \pm 53,0$ & 0,60 \\
\hline Contraste (ml) & $1,9 \pm 1,8$ & $<0,001$ \\
\hline Estadía hospitalaria (días) & & & \\
\hline Tiempo de fluoroscopia & & \\
\hline En 5 pacientes se realizó conversión dela VR a la VF. & & \\
\hline
\end{tabular}

neo en otro territorio vascular no coronario o procedimiento no terapéutico. De esta manera se incluyeron 988 pacientes, 702 con AF y 286 con AR (Figura 1).

El punto final primario del estudio lo constituyen los eventos cardiovasculares mayores (MACCE), que incluye mortalidad de todas las causas, infarto agudo del miocardio (IAM) y complicaciones vasculares mayores (MVC). Dada la naturaleza no aleatorizada del estudio, se hicieron 4 tipos diferentes de análisis de los resultados: (1) población general, (2) una vez excluidos los pacientes con shock cardiogénico o tratados durante maniobras de resucitación cardiopulmonar, (3) una vez excluidos todos los pacientes con STEMI con o sin shock cardiogénico y (4) por último, después de un análisis de poblaciones similares utilizando el método estadístico de propensity score matching (PSM).

Los MVC se definieron si requerían reparación quirúrgica o percutánea por la punción arterial, hematoma retroperitoneal, transfusión de más de 2 unidades y/o síndrome compartimental (SC). Las complicaciones vasculares menores (mvc) se definieron como pseudoaneurismas en el sitio de la punción que no requieren reparación intervencionista y transfusión de 2 o menos unidades de sangre. También se analizaron por separado la muerte, el infarto agudo del miocardio y accidente cerebrovascular. El tiempo de fluoroscopia, la cantidad de sustancia de contraste utilizada y la estadía hospitalaria también fue registrada y comparada entre ambos grupos.

Todos los procedimientos fueron realizados por personal de staff con gran entrenamiento en ambos accesos y utilizando
TABLA 2. Eventos adversos en el hospital en la población global

\begin{tabular}{|l|c|c|c|}
\hline & $\begin{array}{c}\text { Femoral* } \\
\%(n=702)\end{array}$ & $\begin{array}{c}\text { Radial } \\
\%(n=286)\end{array}$ & $p$ \\
\hline Muerte & $1,3(9)$ & $0,3(1)$ & 0,16 \\
\hline IAM con ST (infarto agudo supra ST) & $0,7(5)$ & $0,3(1)$ & 0,44 \\
\hline Stroke & $0,1(1)$ & $0,3(1)$ & 0,49 \\
\hline Complicaciones vasculares mayores (MVC) & $0,4(3)$ & $0,3(1)$ & 0,50 \\
\hline Complicaciones vasculares menores (mVc) & $1,7(12)$ & $0,3(1)$ & 0,12 \\
\hline MACCE (muerte, infarto, MVC) & $2,6(18)$ & $1,4(4)$ & 0,18 \\
\hline
\end{tabular}

técnicas convencionales de intervención. La selección del sitio de acceso del procedimiento terapéutico se realizó a discreción del médico tratante y de las condiciones basales del paciente. La decisión del manejo del sitio de acceso después del procedimiento fue realizada y monitorizada por el médico tratante.

La punción de arteria femoral se realiza con la técnica estándar, con la técnica de Seldinger ${ }^{9}$ modificada, tratando de punzar únicamente sobre la cara anterior de la arteria.

Una vez finalizado el procedimiento y antes de retirar el introductor arterial se realiza en forma sistemática y rutinaria una angiografía digital a 30 grados en la proyección oblicua del cual se realizó el acceso, y según el resultado se procedió con el manejo del introductor: si no había complicaciones relacionadas con la punción femoral, aquel se retiró después de las cuatro horas si el KPTT era $\leq 100$ segundos; si esto no era así, se controlaba a la hora, hasta que se alcanzara el valor referido de KPTT. Se utilizó dispositivo de cierre vascular en los pacientes que se encontraban anticoagulados previamente tanto con NOAC como con acenocumarol o warfarina. En caso de constatarse alguna fuga en el sitio de punción al momento de la angiografía de control ${ }^{9}$, esta era tratada con compresión manual hasta su cierre objetivado por una ecocardiografía del sitio de punción.

El AR fue realizado de acuerdo con técnicas estándar descriptas.

La terapia antiplaquetaria siguió el régimen estándar. Antes del procedimiento, todos los pacientes recibieron una dosis de carga de aspirina $(300 \mathrm{mg}$ ) y clopidogrel $(600 \mathrm{mg})$ o prasugrel $(60$ $\mathrm{mg})$ o ticagrelor $(180 \mathrm{mg})$ a discreción del médico tratante. En el laboratorio de cateterización se administró anticoagulación con un bolo de heparina no fraccionada (75 a $100 \mathrm{U} / \mathrm{kg}$ ) para lograr un tiempo de coagulación activado $\geq 300$ segundos. No se recomendó el uso rutinario de heparina no fraccionada después del procedimiento, a menos que los pacientes necesitaran la colocación de balón de contrapulsación intraaórtico. El inhibidor del receptor de glicoproteína IIb/IIIa se dejó a la discreción del cardiólogo intervencionista, así como la decisión de infundirla posprocedimiento. Después del procedimiento, el uso de la terapia médica estaba a cargo del médico cardiólogo de la unidad coronaria, y la duración de los agentes antiplaquetarios quedó a criterio del médico tratante.

Todos los pacientes firmaron el consentimiento informado de la institución para procedimientos percutáneos y estaban en conocimiento del ingreso de sus datos a un registro.

\section{Estadística}

En las variables continuas, las diferencias entre grupos fueron evaluadas utilizando la prueba t-test a o la prueba de rango de Mann-Whitney. Los datos se expresan como media \pm desvío estándar. Las variables discretas y sus diferencias se expresan como porcentajes, y fueron analizadas con la prueba $\chi 2$ o la exacta de Fisher. El análisis macheado utilizando el PSM se realizó mediante el modelo de regresión logística. Probamos todas las variables disponibles que podrían ser 
TABLA 3, Eventos adversos en el hospital sin shock cardiogénico.

\begin{tabular}{|l|c|c|c|}
\hline & $\begin{array}{c}\text { Femoral* } \\
\%(n=685)\end{array}$ & $\begin{array}{c}\text { Radial \% } \\
(\mathrm{n}=285)\end{array}$ & $\mathrm{p}$ \\
\hline Muerte & $0,14(1)$ & $0(0)$ & 0,39 \\
\hline IAM con ST (infarto agudo supra ST) & $0,72(5)$ & $0,35(1)$ & 0,43 \\
\hline Stroke & $0,14(1)$ & $0,35(1)$ & 0,50 \\
\hline Complicaciones vasculares mayores (MVC) & $0,58(3)$ & $0,35(1)$ & 0,50 \\
\hline Complicaciones vasculares menores (mvc) & $1,7(12)$ & $0,35(1)$ & 0,12 \\
\hline MACCE (muerte, infarto, MVC) & $1,45(10)$ & $1,05(3)$ & 0,34 \\
\hline
\end{tabular}

de relevancia potencial: edad, varones, factores de riesgo cardiovascular (hipertensión, diabetes, dislipidemia, enfermedad cerebrovascular, enfermedad arterial periférica, enfermedad renal crónica, insuficiencia cardíaca y tabaquismo), características angiográficas y de procedimiento, análisis de la lesión, el tipo y la ubicación. El macheo se realizó a través del protocolo de coincidencia 1:1 utilizando el algoritmo de coincidencia, con un ancho de calibre igual a 0,05 de la desviación estándar de la puntuación de propensión, dando 183 pares bien macheados. Prueba antes y después de PSM.

Se realizó un análisis multivariado para encontrar variables independientes de complicaciones cardíacas y vasculares solamente en la población macheada.

Para todos los análisis, se consideró estadísticamente significativa una $\mathrm{p}<0,05$. Todos los datos fueron procesados con SPSS.

\section{RESULTADOS}

En el grupo total de 998 pacientes se observaron amplias diferencias basales demográficas, clínicas y angiográficas. Comparado con el AR, los pacientes incluidos en el AF fueron de más edad ( $\mathrm{p}<0,001)$, hubo mayor cantidad de mujeres $(\mathrm{p}<0,001)$, mayoría con hipertensión arterial $(\mathrm{p}<0,001)$, mayor incidencia de IAM previo $(p<0,05)$, aumento de revascularización previa $(\mathrm{p}=0,005)$, mayor número de pacientes con cirugía de revascularización previa $(, 0,001)$, tratados durante STEMI con angioplastia primaria o de rescate posfibrinolisis $(\mathrm{p}<0,004)$, shock cardiogénico $(\mathrm{p}=0,03)$ y enfermedad de tronco de coronaria izquierda no protegida $(\mathrm{p}=0,023)$. Al contrario, comparados con el AF, los pacientes con AR tuvieron un tratamiento previo más frecuente con inhibidores de IIb/IIIa $(\mathrm{p}=0,009)$ y con mayor obesidad e índice de masa corporal aumentada $(\mathrm{p}<0,001)$ (Tabla1).

La cantidad de sustancia de contraste usada entre los dos grupos y el tiempo de fluoroscopia fue similar, aunque la estadía hospitalaria fue significativamente mayor en AF ( $p<0,001)$ (Tabla 1). No hubo crossover de AF a radial, mientras que $1,7 \%$ inicialmente incluidos en AR se cruzaron a AF. En ningún paciente con AR se utilizó la arteria cubital como vía alternativa. En el grupo general, no hubo diferencias significativas en MACCE o muerte + IAM + accidente cerebrovascular, entre ambos grupos, aunque la muerte y mvc fueron numéricamente más altas en $\mathrm{AF}$ con respecto al AR (1,3 vs. 0,3\% y 1,8 vs. $0,3 \%$; $\mathrm{p}=0,16$ y 0,13 , respectivamente) (Tabla 2 ).

Luego de excluir los pacientes con shock cardiogénico (685 pacientes con AF vs. 285 pacientes con AR) y/o tratados durante un STEMI (584 y 260 pacientes por AF y AR, respectivamente) las diferencias no fueron estadísticamente o numéricamente diferentes (Tablas 3 y 4 ).

En el grupo poblacional macheado después que se realizó el test de PSM (183 pacientes en AF y 183 en AR), donde no se encontraron diferencias significativas en las características clínicas, demográficas o angiográficas de referencia entre
TABLA 4. Eventos adversos en el hospital sin IAM con ST.

\begin{tabular}{|l|c|c|c|}
\hline & $\begin{array}{c}\text { Femoral } \\
\%(n=584)\end{array}$ & $\begin{array}{c}\text { Radial \% } \\
(\mathrm{n}=260)\end{array}$ & $\mathrm{p}$ \\
\hline Muerte & $0,17(1)$ & $0(0)$ & 0,69 \\
\hline IAM con ST (infarto agudo supra ST) & $0,34(2)$ & $0(0)$ & 0,42 \\
\hline Stroke & $0,17(1)$ & $0,38(1)$ & 0,51 \\
\hline Complicaciones vasculares mayores (MVC) & $0,34(2)$ & $0,38(1)$ & 0,51 \\
\hline Complicaciones vasculares menores (mvc) & $1,19(7)$ & $0(0)$ & 0,20 \\
\hline MACCE (muerte, infarto, MVC) & $1,02(6)$ & $0,76(2)$ & 0,36 \\
\hline
\end{tabular}

ambos grupos y solo una tendencia a mayor número de obesos en el grupo radial y pacientes con revascularización previa en el grupo femoral. (Tabla 5), todos los eventos adversos fueron numérica y significativamente similares entre AF y AR: la incidencia de MACCE o muerte ( $p=0,50$ en ambos), MVC y/o mvc ( $p=0,50$ y 0,24 , respectivamente). La estadía hospitalaria, sin embargo, fue significativamente mayor en el AF ( $\mathrm{p}<0,001)$ (Tabla 6). El análisis multivariado de regresión logística para evaluar variables predictivas independientes de MACCE y complicaciones vasculares mayores y menores no encontró ningún predictor significativo entre los 366 pacientes de este grupo.

\section{DISCUSIÓN}

En este registro prospectivo, donde se comparó dos abordajes diferentes en el tratamiento percutáneo coronario, podemos observar que en este grupo experimentado de operadores con ambas técnicas no se encontraron diferencias significativas en eventos cardiovasculares mayores en el análisis general como luego de excluir grupos de alto riesgo y en el grupo de análisis con PSM.

En el grupo general hubo una tendencia numérica de más eventos adversos mayores y de sangrado con el AF comparado con el AR, pero esto estaría relacionado a mayores morbilidades basales de los pacientes con AF.

La ausencia de diferencias en esta serie entre ambos accesos puede deberse a varias circunstancias. Primero, a que los actuales resultados comparativos entre ambos grupos en estudios aleatorizados y registros no son similares y en algunos casos los resultados son contradictorios. Segundo, a la larga experiencia del grupo de operadores en esta vía de acceso, que incluye un protocolo de trabajo de angiografía sistemática al finalizar el procedimiento en sustracción digital en todos los pacientes, que es evaluada cuidadosamente por posibles complicaciones y así como a su pronta resolución ${ }^{9}$. Creemos que todos estos factores combinados y/o aislados fueron determinantes para el bajo número de complicaciones vasculares observados en este reporte.

En los últimos años se publicaron varios estudios aleatorizados comparativos entre ambos accesos con algunos resultados favorables al AR, aunque no fueron uniformes.

El estudio RIVAL, que incluyó pacientes con síndrome coronario agudo, no encontró diferencias significativas en muerte, infarto y accidente cerebrovascular entre los dos grupos $(3,2 \%$ en el grupo radial y $3,2 \%$ en el grupo femoral), pero con aumento de hematomas en el grupo femoral ${ }^{10}$. El RIFLE-STEACS, donde aleatorizaron pacientes con STEMI, mostró una diferencia significativa en la mortalidad cardíaca (9,2\% en el grupo femoral vs. 5,2\% en el grupo radial $)^{11}$; sin embargo, la mortalidad en el AF de este estudio está muy por encima de la mortalidad reportada en similar grupo de pacientes en nuestro país por el registro RA$\mathrm{DAC}^{12}$. Es de notar que en los primeros estudios observacionales y aleatorizados con stents en STEMI que utilizaron el 
TABLA 5. Características basales clínicas y angiográficas. Población macheada.

\begin{tabular}{|l|c|c|c|}
\hline & $\begin{array}{c}\text { Femoral } \\
\%(\mathrm{n}=183)\end{array}$ & $\begin{array}{c}\text { Radial } \\
(\mathrm{n}=183)\end{array}$ & $\mathrm{p}$ \\
\hline Mujeres & $11,5(21)$ & $11,5(21)$ & 1,00 \\
\hline Edad (años) & $63,6 \pm 9,8$ & $63,6 \pm 9,8$ & 0,97 \\
\hline Hipertensión arterial & $68,9(126)$ & $66,1(121)$ & 0,57 \\
\hline Dislipemia & $70,5(129)$ & $68,9(126)$ & 0,73 \\
\hline Tabaquista & $19,1(35)$ & $22,4(41)$ & 0,43 \\
\hline Diabetes mellitus & $23,5(43)$ & $24,0(44)$ & 0,90 \\
\hline Antecedentes heredofamiliares & $15,3(28)$ & $13,1(24)$ & 0,54 \\
\hline IAM previo & $26,2(48)$ & $23,0(42)$ & 0,46 \\
\hline Revascularización previa & $34,4(63)$ & $25,1(46)$ & 0,059 \\
\hline Enfermedad vascular periférica & $6,0(11)$ & $7,1(13)$ & 0,67 \\
\hline Stroke & $1,6(3)$ & $2,7(5)$ & 0,36 \\
\hline Mala función ventricular & $7,1(13)$ & $5,5(10)$ & 0,51 \\
\hline Insuficiencia renal crónica & $4,9(9)$ & $3,3(6)$ & 0,30 \\
\hline BMI>29 & $19,1(35)$ & $27,3(50)$ & 0,06 \\
\hline Síndrome coronario agudo & $90,2(165)$ & $88,5(162)$ & 0,61 \\
\hline IAM con ST & $8,7(16)$ & $8,7(16)$ & 1,00 \\
\hline Enfermedad de múltiples vasos & $39,3(72)$ & $38,8(71)$ & 0,91 \\
\hline $\begin{array}{l}\text { Enfermedad de tronco de coronaria } \\
\text { izquierda no protegida }\end{array}$ & $6,0(11)$ & $4,4(8)$ & 0,48 \\
\hline $\begin{array}{l}\text { Tratamiento previo con inhibidores } \\
\text { de la 2PY12 }\end{array}$ & $17,5(32)$ & $24,0(44)$ & 0,12 \\
\hline Tipo de inhibidores de la 2PY12 & $20,8(38)$ & $10,4(19)$ & 0,006 \\
\hline Prasugrel & $39,9(73)$ & $58,5(107)$ & $<0,001$ \\
\hline Ticagrelor & $38,8(71)$ & $30,6(56)$ & 0,10 \\
\hline Clopidogrel & $17,6(32)$ & $24(44)$ & 0,12 \\
\hline Inhibidores de Ilb/llla & $1,78 \pm 0,96$ & $1,73 \pm 0,90$ & 0,61 \\
\hline Stents & 222 & 217 & 0,80 \\
\hline BMS (n) & 100 & 0,80 \\
\hline DES (n) & $179 \pm 50$ & 0,47 \\
\hline Contraste (ml) & $2,0 \pm 1,4$ & $<0,001$ \\
\hline Estadía hospitalaria (días) & & \\
\hline
\end{tabular}

AF la mortalidad reportada fue mucho menor ${ }^{13-15}$ que la observada en el estudio RIFLE ${ }^{11}$. De hecho, el estudio GRA$\mathrm{MI}^{14}$ reportó 3,8\% de mortalidad hospitalaria, que fue del $2,1 \%$ cuando se excluyeron pacientes con shock cardiogénico. El estudio STEMI-Radial no encontró diferencias significativas en la mortalidad a los 30 días $(2,3 \%$ vs. $3,1 \%$ para AR y $\mathrm{AF}$, respectivamente; $\mathrm{p}=0,64$ ), aunque en los eventos combinados incluyendo sangrado fue favorable al grupo radial ${ }^{16}$, al igual que el estudio MATRIX ${ }^{17}$. Sin embargo, el más reciente estudio aleatorizado en pacientes con STEMI para valorar la seguridad y eficacia de ambos accesos, conducido en Canadá y presentado en la sesión del American College of Cardio$\operatorname{logy} 2019^{18}$, no dio diferencias entre uno u otro grupo en mortalidad ( $1,5 \%$ con AR y $1,3 \%$ con AF), ni complicaciones vasculares o cardíacas durante el período hospitalario y a 30 días. Por último, en el trabajo más reciente de comparación entre ambos accesos en pacientes con oclusiones totales crónicas, los autores no encontraron diferencias en eventos cardiovasculares mayores entre los grupos y solo un aumento de sangrado en el grupo femoral, pero hay que remarcar que el grupo radial tuvo alta proporción de crossover y además las oclusiones eran claramente menos complejas y en pacientes más jóvenes ${ }^{19}$ que en el grupo AF.

Los resultados de los metaanálisis también arrojan datos discordantes. Así, un metaanálisis con estas técnicas en pacientes con infarto agudo del miocardio sobre 21 ensayos aleatorizados y registros que incluyeron 8534 pacientes $^{20}$, estos presentaron una reducción significativa de los eventos cardiovasculares mayores y sangrado a favor de la técnica AR, sin diferencia significativa en el tiempo puerta-balón o de la fluoroscopia; pero otro metaanálisis posterior de Sukhchain
TABLA 6. Eventos adversos en el hospital de la población macheada.

\begin{tabular}{|l|c|c|c|}
\hline & $\begin{array}{c}\text { Femoral* } \\
\%(n=183)\end{array}$ & $\begin{array}{c}\text { Radial } \\
\%(n=183)\end{array}$ & $p$ \\
\hline Muerte & $0,5(1)$ & $0,0(0)$ & 0,50 \\
\hline IAM con ST (infarto agudo supra-ST) & $1,1(2)$ & $0,0(0)$ & 0,24 \\
\hline Stroke & $0,0(0)$ & $0,5(1)$ & 0,50 \\
\hline Complicaciones vasculares mayores (MVC) & $0,5(1)$ & $0,5(1)$ & 1,00 \\
\hline Complicaciones vasculares menores (mvc) & $0,5(1)$ & $0,0(0)$ & 0,18 \\
\hline MACCE (muerte, infarto, MVC) & $1,6(3)$ & $1,1(2)$ & 0,50 \\
\hline
\end{tabular}

et al. ${ }^{21}$, que analizó a 14 ensayos con un pool de paciente de 3758 e incluyó solo pacientes con STEMI, halló diferencias significativas a favor del procedimiento femoral en cuanto al tiempo de fluoroscopia, tiempo puerta-balón y nivel de crossover de AR a AF con similar mortalidad. Finalmente, un tercer metaanálisis, de Ferrante et $\mathrm{al}^{22}$, que incluyó 24 ensayos y enroló 22.842 pacientes, halló diferencias significativas en todos los eventos, incluyendo la mortalidad de todas las causas así como el aumento del sangrado a favor del AR. En nuestro medio, recientemente, se reportó una experiencia de un solo centro, observacional, retrospectiva, con resultados favorables a los pacientes tratados por vía radial. Sin embargo, los autores de este registro compararon poblaciones muy diferentes y no realizaron un análisis de resultados entre poblaciones $m a$ cheadas, por lo que de este estudio ${ }^{23}$ no se pueden extraer conclusiones sobre cuál vía de acceso fue la más adecuada.

Otro tema remarcable es que, en EE.UU., según el último reporte del Cath Lab PCI Registry del National Cardiovascular Data Registry (NCDR), la técnica radial todavía se encontraba por debajo del $30 \%$, aunque esto se pudo haber incrementado en los últimos tiempos. En este registro del NCDR, cuando analizaron los 9494 pacientes que presentaron STEMI y habían sido fibrinolizados y derivados a angioplastia de rescate, la ruta de elección fue la femoral en un $84 \%$ de los pacientes, que después de realizar propensity-matched analyses no halló diferencias en la mortalidad ${ }^{24}$.

Un tema no menor es el de la radiación, como se vio en el subestudio de RIVAL ${ }^{25}$, donde era mayor en el AR que en el AF, y se vio aún más aumentada en la técnica con radial izquierda. En el registro que estamos reportando acá no hubo diferencias significativas en tiempo de fluoroscopia entre ambos accesos, lo que junto con la baja incidencia de crossover de AR a AF, nos sugiere de la experiencia de los operadores con ambas técnicas.

Se ha mencionado en algún momento que la técnica de AF podría estar cayendo en desuso, pero esto ha tenido un cambio drástico en los últimos diez años debido al ingreso y auge del implante percutáneo de válvula aórtica así como al uso de dispositivos de soporte cardiocirculatorio ${ }^{26-28}$,

Debido a todos estos aspectos creemos que la vía femoral es y seguirá siendo una vía de elección en muchos pacientes, principalmente aquellos con necesidad de procedimientos percutáneos coronarios complejos. Sin embargo, es mandatorio que ambos accesos deban ser parte necesaria del entrenamiento rutinario de fellows en cardiología intervencionista.

\section{LIMITACIONES DEL ESTUDIO}

Este estudio tiene varias limitaciones: se trata de un estudio observacional, no aleatorizado, que incluyó un grupo mayor de pacientes con técnica femoral que radial. No sabemos claramente el motivo de la selección de cada vía de acceso; de hecho, los pacientes en el grupo femoral tenían un mayor riesgo basal global. El tamaño de la muestra para reali- 
zar un PSM es pequeño y no podemos descartar que existan factores confundidores. Además, al ser un servicio de entrenamiento en cardiología intervencionista y el cuidado posintervención del paciente realizado por médicos residentes en tiempo completo, los resultados en AF podrían no ser extrapolable a otros servicios que no cuenten con esos cuidados. El seguimiento fue a 30 días, y complicaciones inherentes a los procedimientos aparecidas luego de ese período no están incluidas, aunque, la gran mayoría de ellas aparecen descriptas dentro de ese período.

Finalmente, el análisis del sangrado se realizó sobre la base de complicaciones sanguíneas mayores y no sobre scores de sangrado, aunque hay un gran número de definiciones en este aspecto por lo cual la estandarización es difícil. ${ }^{29}$

\section{BIBLIOGRAFÍA}

1. Sones FM Jr. Acquired heart disease: symposium on present and future of cineangiocardiography. Am J Cardiol 1959;3:710.

2. Gruntzig A. Transluminal dilatation of coronary-artery stenosis. Lancet 1978 Feb 4;1(8058):263.

3. Rodríguez AE, Díaz R, Zuffardi E, Navarro P, de la Fuente LM. Angioplastia transluminal percutánea coronaria. Rev Arg Cardiol 1982;50(2):78-91.

4. Campeau L. Percutaneous radial artery approach for coronary angiography. Cathet Cardiovasc Diagn 1989;16:3-7.

5. Kiemeneij F, Laarman GJ, de Melker E. Transradial artery coronary angioplasty. Am Heart J 1995 Jan;129(1):1-7.

6. Kiemeneij F, Laarman GJ, Odekerken D, Slagboom T, van der Wieken R. A randomized comparison of percutaneous transluminal coronary angioplasty by the radial, brachial and femoral approaches: the access study. J Am Coll Cardiol 1997 May;29(6):1269-75.

7. Neumann FJ, Sousa-Uva M, Ahlsson A, et al. 2018 ESC/EACTS Guidelines on myocardial revascularization. Eurolntervention 2019 Feb 20;14(14):1435-534.

8. Dehmer GJ, Weaver D, Roe MT, et al. A contemporary view of diagnostic cardiac catheterization and percutaneous coronary intervention in the United States: areportfrom the Cath PCI Registry of the National Cardiovascular Data Registry, 2010 through June 2011.J Am Coll Cardiol 2012;60:2017-31.

9. Alonso M, Tascón J, Hernández F, et al. Complications with femoral access in cardiac catheterization. Impact of previous systematic femoral angiography and hemostasis with VasoSeal-ES collagen plug. Rev Esp Cardiol 2003 Jun:56(6):569-77.

10. JollySS, YusufS, Cairns J, et al. Radial versus femoral access for coronary angiography and intervention in patients with acute coronary syndromes (RIVAL): a randomised, parallel group, multicentre trial. Lancet 2011;377:1409-20.

11. RomagnoliE, Biondi-ZoccaiG, SciahbasiA, etal. Radial versus femoral randomized investigation in ST-segmentelevation acute coronary syndrome: the RIFLE-STEACS (Radial Versus Femoral Randomized Investigation in ST-Elevation Acute Coronary Syndrome) study. J Am Coll Cardiol 2012;60:2481-9.

12. Rubilar B, Martin R, Coroleu S, etal. Resultados intrahospitalarios dela angioplastia coronaria en octogenarios. Subestudio del Registro Argentino de Angioplastia Coronaria (RAdAC). Revista Argentina de Cardioangiología 2015;(04):0180-6.

13. Rodríguez A, Fernández M, Santaera O et al Coronary stenting in patients undergoing percutaneous transluminal coronary angioplasty during acute myocardial infarction. Am J Cardiol 1996;77:685-9.

14. Rodríguez A, Bernardi V, Fernández $M$, et al. In-hospital and late results of coronarystents versus conventional balloon angioplasty in acute myocardial infarction (GRAMI trial). Gianturco-Roubin in AcuteMyocardial Infarction. Am J Cardiol 1998 Jun 1;81(11):1286-91.

15. Antoniucci D, Santoro GM, Bolognese L, Valenti R, Trapani M, Fazzini PF. A clinical trial comparing primary stenting of the infarct-related artery with optimal primary angioplasty for acute myocardial infarction: results from the Florence Randomized Elective Stenting in Acute Coronary Occlusions (FRESCO) trial. J Am Coll Cardiol 1998;31 (6):1234-9.

\section{CONCLUSIONES}

En este registro prospectivo se observó que la utilización tanto de los accesos femoral como radial estuvo asociada a una baja incidencia de complicaciones hospitalarias y a 30 días.

No hubo diferencia significativa en mortalidad y complicaciones vasculares entre los dos grupos en el análisis de los 988 pacientes de la población global, aunque numéricamente las complicaciones vasculares menores y la mortalidad fueron mayores en el AF.

En el análisis de resultados de los 366 pacientes con características basales similares, no se observaron diferencias numéricas ni significativas entre ambos grupos incluyendo complicaciones vasculares menores.

16. Bernat I, Horak D, Stasek J, et al. ST-segment elevation myocardial infarction treated by radial or femoral approach in a multicenter randomized clinical trial: the STEMI-RADIAL trial. J Am Coll Cardiol 2014;63:964-72.

17. Valgimigli M, Gagnor A, Calabró P, et al. Radialversus femoral access in patients with acute coronary syndromes undergoing invasive management: a randomized multicentre trial. Lancet 2015;385:2465-76.

18. Le May M, et al. SAFARI-STEMI: Similar 30-Day Mortality For Radial vs. Femoral Access PCI. American College of Cardiology Annual Scientific Session (ACC 2019), New Orleans, LA, March 18, 2019

19. Tajti P, Alaswad K, Karmpaliotis D and et al. Procedural Outcomes of Percutaneous Coronary Interventions for Chronic Total Occlusions Via the Radial Approach:Insights From an International Chronic Total Occlusion Registry. JACC Cardiovasc Interv 2019 Feb 25;12(4):346-58.

20. Jang JS, Jin HY, Seo JS, et al. The transradial versus the transfemoral approach for primary percutaneous coronary intervention in patients with acute myocardial infarction: a systematic review and meta-analysis. Eurolntervention 2012 Aug;8(4):501-10.

21. Singh S, Singh M, Grewal N, Khosla S. The fluoroscopy time, door to balloon time, contrast volume use and prevalence of vascular access site failure with transradial versus transfemoral approach in ST segment elevation myocardial infarction: A systematic review \& meta-analysis. Cardiovasc Revasc Med 2015 Dec;16(8):491-7.

22. Ferrante G, Rao SV, Jüni P, et al. Radial Versus Femoral Access for Coronary Interventions Across the Entire Spectrum of Patients With Coronary Artery Disease: A Meta-Analysis of Randomized Trials. JACC Cardiovasc Interv 2016 Jul 25:9(14):1419-34.

23. Eficiencia y seguridad del acceso radial versus el acceso femoral en la angioplastia coronaria. Abud MA, Padilla LT, Pedernera GO, et al. Rev Argent Cardiol 2019;87:26-33

24. Kadakia MB, RaoSV, McCoy L, et al. Transradial Versus Transfemoral Access in Patients Undergoing Rescue Percutaneous Coronary Intervention After Fibrinolytic Therapy. JACC Cardiovasc Interv 2015 Dec 21;8(14):1868-76.

25. Jolly SS, Cairns J, Niemela K, et al. Effect of radial versus femoral access on radiation dose and the importance of proceduralvolume: a substudy of the multicenter randomized RIVAL trial. JACC Cardiovasc Interv 2013:6:258-66.

26. Arora S, Vaidya SR, StrasslePD, et al. Meta-analysis of transfemoral TAVR versus surgical aortic valve replacement. Catheter Cardiovasc Interv 2018 Mar 1;91(4):806-12

27. Makdisi G, Wang IW. Extra Corporeal Membrane Oxygenation (ECMO) review of a lifesaving technology. J Thorac Dis 2015 Jul;7(7):E166-76.

28. CheraHH,NagarM, Chang NL, etal.Overview of Impella andmechanicaldevices in cardiogenic shock. Expert Rev Med Devices. 2018 Apr;15(4):293-299.

29. Généreux P, Giustino G, Witzenbichler B, et al Incidence, Predictors, and Impact of Post-Discharge Bleeding After Percutaneous Coronary Intervention. J Am Coll Cardiol. 2015 Sep 1;66(9):1036-45. doi: 10.1016/j. jacc.2015.06.1323. 